\title{
Projective Synchronization of a 3-D Chaotic System with Quadratic and Quartic Nonlinearities.
}

\section{Babatunde A. Idowu ${ }^{1}$, Kehinde, S. Oyeleke ${ }^{2}$, Cornelius O. Ogabi ${ }^{1}$, Olasunkanmi I. Olusola ${ }^{2}$}

${ }^{1}$ Department of Physics, Faculty of Science, Lagos State University, Nigeria.

${ }^{2}$ Nonlinear Dynamics Research Group, Department of Physics, University of Lagos, Akoka, Lagos, Nigeria.

\section{Correspondence}

Babatunde A. Idowu

babatunde.idowu@lasu.edu.ng

Phone: 08023029899

\section{Abstract:}

Introduction: Chaos is a state of dynamical systems whose apparently random states of disorder and irregularities are often governed by deterministic laws that are highly sensitive to initial conditions. In this work, the projective synchronization of two identical three-dimensional chaotic system with quadratic and quartic nonlinearities was considered as well as the equilibrium and stability analysis of the system. The projective synchronization with same and different scaling factors was carried out in order to establish its synchronization.

Aim: :To achieve projective synchronization of two identical three-dimensional chaotic system with quadratic and quartic nonlinearities synchronizing to a scaling factor and also present the equilibrium and stability analysis of the system.

Methods: We employed the adaptive synchronization technique to achieve projective synchronization of the system (master and slave) with different scaling factors, $\beta$ and the fourth-order Runge-Kutta algorithm was used for numerical solutions.

Results: In this work, the projective synchronization of two identical threedimensional systems with quadratic and quartic nonlinearities was achieved with the same and different scaling factors, $\beta$. The equilibrium and stability analysis of the system was also presented.

Conclusion: The investigated projective synchronization behaviour of two identical three-dimensional system with two nonlinearities (quadratic and quartic) was achieved for cases where the scaling factor is the same and when different. This shows that projective synchronization can be achieved for systems with varying nonlinearities even when the scaling factor is different and this suggests its use in communication using chaotic wave forms as carriers, perhaps with a view to securing communication.

Keywords: Projective Synchronization, nonlinearities, scaling factors, RungeKutta, Quadratic and quartic nonlinearities.

This is an open access article under the terms of the Creative Commons Attribution License, which permits use, distribution and reproduction in any medium, provided the original work is properly cited. (C) 2018 The Authors. Journal of Research and Reviews in Science - JRRS, A Publication of Lagos State University 


\section{INTRODUCTION}

Chaos synchronization is an extension of the concept of chaos control. Nowadays, the control and synchronization of the chaotic systems have received a lot of attention by researchers because of its unpredictable complex behaviour. The ultimate objective of chaos synchronization is to design a feedback controller for the follower chaotic system such that the drive system tracks the trajectories of the driven chaotic system as time goes to infinity. However, the challenges occur when the chaotic systems are exposed to some uncertainty, unknown system parameters and have different initial values. Then, some actions have to be taken in order to stabilize and to improve synchronization. In 1963, Lorenz discovered the famous Lorenz chaotic system [1]. After that, chaotic systems have been researched extensively, such as the Lu system $[2,3,4]$ the Chen system [5] and the Rössler system [6] new chaotic finance system [7] and so on.

Chaotic systems must have one positive Lyapunov exponents while Hyperchaotic systems must have at least two positive Lyapunov exponents, and the dimension must be four or more [8]. Chaotic systems are suitable for some engineering applications, such as chemical reactions, electric circuits [9], cryptography [10, 11] and fluid dynamics and secure communication [12, 13, 14].

Chaos synchronization is another fascinating concept. Pecora and Carroll proposed a drive-response chaotic synchronization scheme in $1990[15,16]$ and realized the synchronization of two chaotic systems in the circuit, which promoted the theoretical study of chaotic synchronization and chaos control. Since then, many effective chaotic synchronization methods have emerged, such as complete synchronization $[17,18]$ generalized synchronization [19], phase synchronization [20], lag synchronization [21, 22, 23], projective synchronization $[24,25]$ anticipating synchronization [26] and exponential synchronization $[27,28]$. In recent years, the synchronization of chaotic fractional differential systems [29, 30, 31] has attracted more and more attention because of its potential applications in secure communication and control processes [32, 33, 34, 35].

The research on projective synchronization [36] has received extensive attention from researchers in recent years. Projective synchronization suffice that under certain conditions, the output of the coupled drive system and the response system state is not only phase locked, but the amplitude of each corresponding state also evolve according to a certain scale factor relationship. The method has been observed and discussed in coupled integer order chaotic systems $[37,38,39,40]$.

Modified projective synchronization, was considered by Li [41, 42] in which the response of synchronized dynamical states can synchronize up to a constant matrix. The modified projective synchronization was an extension of generalized projective synchronization.

There is also fuzzy adaptive controller for achieving an appropriate generalized projective synchronization (GPS) of two incommensurate fractional-order chaotic systems. The master system and the slave system, are assumed to be with non-identical structure, exter- nal dynamical disturbances, uncertain models and distinct fractionalorders. The adaptive fuzzy systems are used for estimating some unknown nonlinear functions, see [43]. In achieving synchronization many methods have been proposed such as active control [44, $45,46,47,48]$, backstepping $[49,50]$, adaptive backstepping [51, $52,53,54]$, sliding mode, and so on.

The other parts of the article is organized as follows: In section 2 , a new three-dimensional system with two nonlinearities is constructed and the dynamical behaviours of the system are discussed, such as attractor, dissipativity and equilibrium points. In section 3 , the projective synchronization scheme of the new three-dimensional system with two nonlinearities is designed and some numerical simulations are completed. In section 4, some conclusions.

\section{SYSTEM DESCRIPTION, EQUILIBRIUM AND STABILITY}

\subsection{Systems Description}

Vaidyanathan et al. $[55,56,57]$ propose a new three-dimensional system with two nonlinearities given by the following nonlinear differential equation, (1):-

$$
\begin{aligned}
& \dot{x_{1}}=a\left(x_{2}-x_{1}\right) \\
& \dot{x_{2}}=x_{1} x_{3} \\
& \dot{x_{3}}=50-b x_{1}^{4}-c x_{3}
\end{aligned}
$$

where $x_{1}, x_{2}, x_{3}$ are the states of the system and $a, b, c$ are positive parameters, and control the dynamics of the system. With system (1) describes a self-excited chaotic attractor phase space plot as shown in figure 1 . The initial condition is taken as $(2.6,3.7,1.4)$. Also, the slave system is described by the controlled chaotic system

$$
\begin{aligned}
& \dot{y_{1}}=a\left(y_{2}-y_{1}\right)+u_{1} \\
& \dot{y_{2}}=y_{1} y_{3}+u_{2} \\
& \dot{y_{3}}=50-b y_{1}^{4}-c y_{3}+u_{3}
\end{aligned}
$$

Where $y_{1}, y_{2}, y_{3}$ are the states and $u_{1}, u_{2}, u_{3}$ are active controllers to be designed.

The divergence of system (1) is in the form

$$
\nabla V=\frac{d \dot{x_{1}}}{d x_{1}}+\frac{d \dot{x_{2}}}{d x_{2}}+\frac{d \dot{x_{3}}}{d x_{3}}
$$

which is $-(a+0+c)=(3+0+1)=-4<0$ 


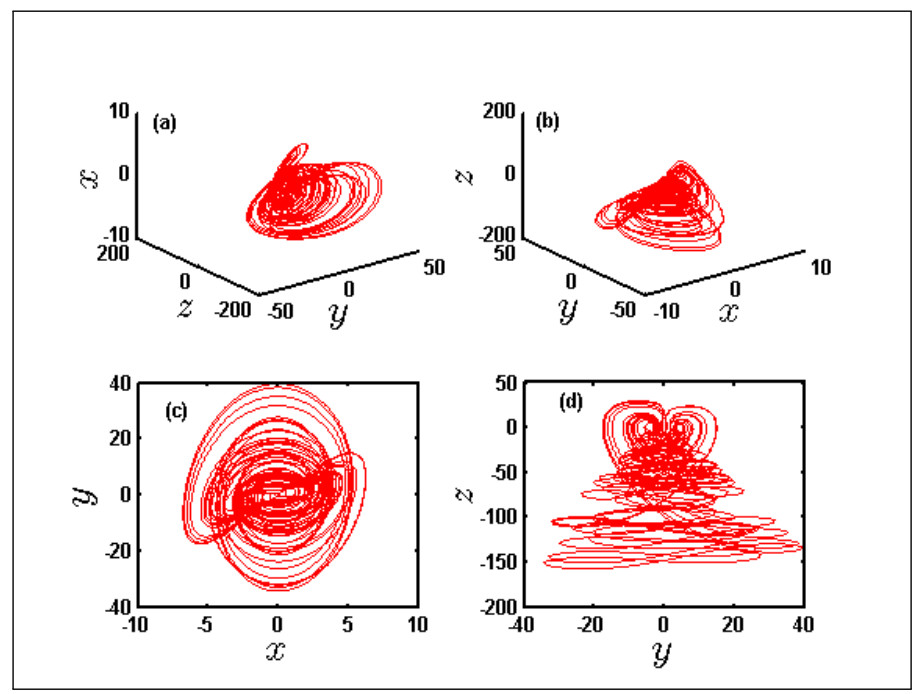

Figure 1: Phase Portrait of system (1): (a), $\left(x_{2}, x_{3}, x_{1}\right)$ plane; (b), $\left(x_{1}, x_{2}, x_{3}\right)$ plane; $(\mathrm{c}),\left(x_{1}, x_{2}\right)$ plane; $(\mathrm{d}),\left(x_{2}, x_{3}\right)$ plane.

\subsection{Equilibrium and Stability Analysis}

The equilibrium of the system (1) can be obtained by solving the following equations

$$
\begin{aligned}
a\left(x_{2}-x_{1}\right) & =0 \\
x_{1} x_{3} & =0 \\
50-b x_{1}^{4}-c x_{3} & =0
\end{aligned}
$$

It is easy to find that system (4) has only one trivial equilibrium point . The Jacobian matrix is given by

$$
J=\left[\begin{array}{ccc}
-a & a & 0 \\
50 & 0 & 0 \\
0 & 0 & -c
\end{array}\right]
$$

Taking $x_{1}=0$, then $x_{2}=0$ and $x_{3}=50$ The eigenvalues of the Jacobian matrix $J$ are: $\lambda_{1}=-13.8390, \lambda_{2}=10.8390, \lambda_{3}=$ -1.0000 The eigenvalues are real and have opposite signs, this shows that the equilibrium of the system is a saddle point.

\section{METHODS:}

\subsection{Projective synchronization with the same scal- ing factors}

Our objective is to find suitable controllers $u_{i},(i=1,2,3)$ to ensure the drive system (1) and the response system (2) approach projective synchronization with a scaling factor, $\beta$.The projective synchronization error vector is defined by $e_{i}=x_{i}-\beta y_{i},(i=1,2,3)$.
Then the error dynamics is obtained as

$$
\begin{aligned}
& \dot{e_{1}}=a\left(e_{2}-e_{1}\right)-\beta u_{1} \\
& \dot{e_{2}}=x_{1} x_{3}-\beta y_{1} y_{3}-\beta u_{2} \\
& \dot{e_{3}}=50(1-\beta)-b\left(x_{1}^{4}-\beta y_{1}^{4}\right)-c e_{3}-\beta u_{3}
\end{aligned}
$$

With different initial conditions, trajectories of the systems (1) and (2) without controllers will diverge exponentially due to the butterfly effect. Nevertheless, when suitable controllers are designed, the two chaotic systems will approach projective synchronization for any initial conditions.

In order to achieve this objective, we choose the following controllers

$$
\begin{aligned}
& u_{1}=\frac{1}{\beta}\left[a\left(e_{2}-e_{1}\right)+e_{1}\right] \\
& u_{2}=\frac{1}{\beta}\left[x_{1} x_{3}-\beta y_{1} y_{3}+e_{2}\right] \\
& u_{3}=\frac{1}{\beta}\left[50(1-\beta)-b\left(x_{1}^{4}-\beta y_{1}^{4}\right)-c e_{3}+e_{3}\right]
\end{aligned}
$$

Systems (1) and (2) will achieve projective synchronization asymptotically with the controllers (7) with a scaling factor.

Proof. Choose the following Lyapunov function

$$
V(e)=e^{T} P e=\frac{1}{2}\left(e_{1}^{2}+e_{2}^{2}+e_{3}^{2}\right)
$$

Where, $P=\operatorname{dia}[1,1,1]$ The derivative of the Lyapunov function $V(e)$ with respect to time is:

$$
\begin{aligned}
\dot{V}(e) & =e_{1} \dot{e_{1}}+e_{2} \dot{e_{2}}+e_{3} \dot{e_{3}} \\
& =e_{1}\left(a e_{2}-a e_{1}-\beta u_{1}\right)+e_{2}\left(x_{1} x_{3}-\beta y_{1} y_{3}-\beta u_{2}\right) \\
& +e_{3}\left(50(1-\beta)-b\left(x_{1}^{4}-\beta y_{1}^{4}\right)-c e_{3}-\beta u_{3}\right)
\end{aligned}
$$

Substitute equation (7) into equation (9)

$$
\begin{aligned}
& \dot{V}(e)=e_{1}\left(-e_{1}\right)+e_{2}\left(-e_{2}\right)+e_{3}\left(-e_{3}\right) \\
& \dot{V}(e)=-\left(e_{1}^{2}+e_{2}^{2}+e_{3}^{2}\right) \\
& \dot{V}(e)<0
\end{aligned}
$$

Therefore, $\dot{V}(e)$ is negative definite. And according to the Lyapunov stability theory, the error dynamical system (6) is asymptotically stable at the origin. Therefore, projective synchronization between the system (1) and (2) is achieved with the controllers (7). The proof is now complete.

\subsubsection{Result discussion and output of results}

Next, we perform numerical simulations to show the feasibility and effectiveness of the designed controller. Choose the scaling factor, $\beta=5$. The 4th order Runge-Kutta method is employed to integrate the differential equations. The initial conditions of the drive 
system and the response system are $(2.6,3.7,1.4)$ and $(1.4,5.9,2.1)$, respectively. The synchronization of systems (1) and (2) is achieved using the controllers (7). The result shows the state variable moves chaotically with time when the controller is de-activated. This shows that these systems depend sensitively on initial condition in the absence of controller, which is a major character of a chaotic system. We depict in Figure 2 that the variable errors do not synchronize with time in the absence of controllers but when the controllers are introduced at $t \geqslant 5.5$ the variable errors synchronize with time. This is confirmed by the synchronization quality $e$, given by $e=\sqrt{\left(e_{1}^{2}+e_{2}^{2}+e_{3}^{2}\right)}$ as shown in Figure 3 .

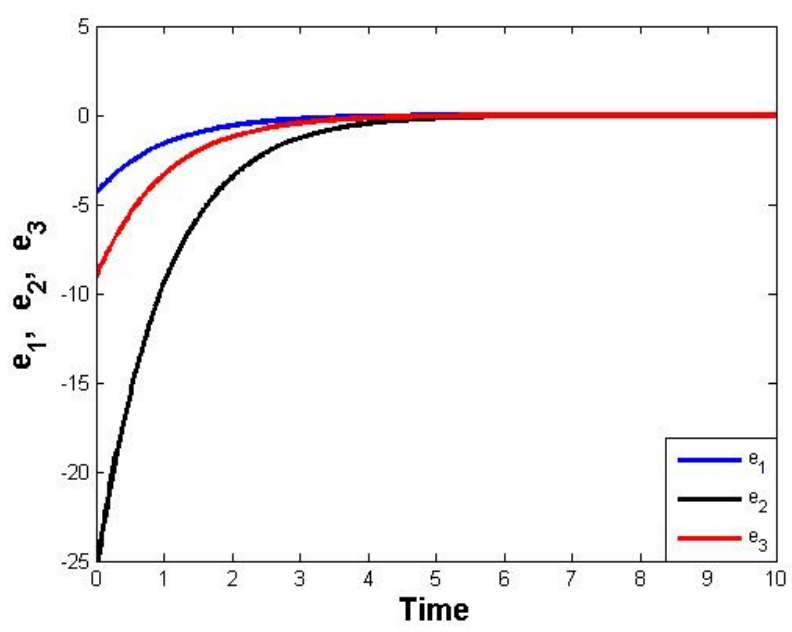

Figure 2: Error dynamics between systems (11) and (2) with the controllers deactivated for $0<t=5.5$ and activated for $t \geqslant 5.5$.

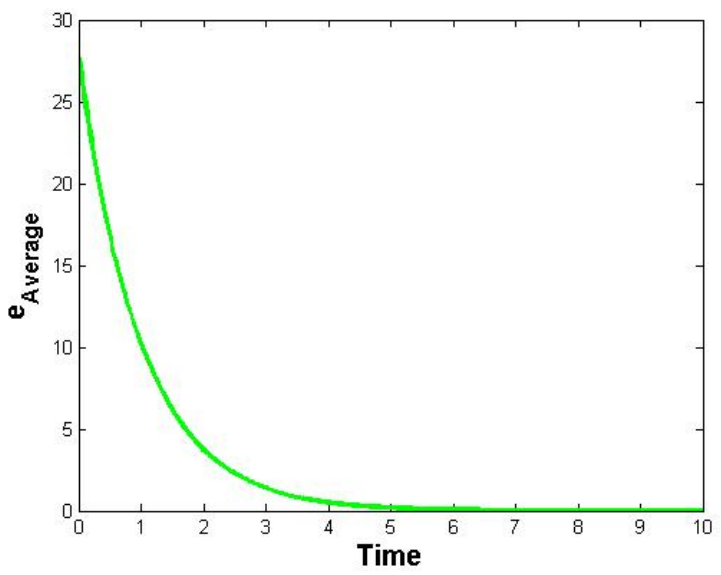

Figure 3: Synchronization quality between systems(1) and (2). ent scaling factors which implies that the three state variables of the drive system are in proportion to that of the response system with three different scaling factors $\beta_{1}, \beta_{2}, \beta_{3}$ respectively. In other word, there exists a constant matrix $\beta=\operatorname{dia}\left(\beta_{1}, \beta_{2}, \beta_{3}\right)$ such that $\lim _{t \rightarrow \infty}|x-\beta y|=0$ This synchronization form called 'modified projective synchronization' in $[58,59,60]$ which has been considered in chaotic systems . We choose exactly the same master-slave system described in the last section, and define the error vectors as $e_{i}=x_{i}-\beta_{i} y_{i},(i=1,2,3)$. Hence, the error dynamical system is obtained as

$$
\begin{aligned}
& \dot{e_{1}}=a\left(x_{2}-\beta_{1} y_{2}\right)-a e_{1}-\beta_{1} u_{1} \\
& \dot{e_{2}}=x_{1} x_{3}-\beta_{2} y_{1} y_{3}-\beta_{2} u_{2} \\
& \dot{e_{3}}=50\left(1-\beta_{3}\right)-b\left(x_{1}^{4}-\beta_{3} y_{1}^{4}\right)-c e_{3}-\beta_{3} u_{3}
\end{aligned}
$$

Our focus is to design suitable controllers to achieve projective synchronization with different scaling factors between the drive systems (1) and the response system (2). Choosing the control functions $u_{i},(i=1,2,3)$ as

$$
\begin{aligned}
& u_{1}=\frac{1}{\beta_{1}}\left[a\left(x_{2}-\beta_{1} y_{2}\right)-a e_{1}+e_{1}\right] \\
& u_{2}=\frac{1}{\beta_{2}}\left[x_{1} x_{3}-\beta_{2} y_{1} y_{3}+e_{2}\right] \\
& u_{3}=\frac{1}{\beta_{3}}\left[50\left(1-\beta_{3}\right)-b\left(x_{1}^{4}-\beta_{3} y_{1}^{4}\right)-c e_{3}+e_{3}\right]
\end{aligned}
$$

Proof: Systems (1) and (2) will achieve projective synchronization asymptotically with the controllers (11) with different scaling factors. Proof: Choose the following Lyapunov function

$$
V(e)=e^{T} P e=\frac{1}{2}\left(e_{1}^{2}+e_{2}^{2}+e_{3}^{2}\right)
$$

Where $P=\operatorname{dia}[1,1,1]$ The derivative of the Lyapunov function $V(e)$ with respect to time is

$$
\begin{aligned}
\dot{V}(e) & =e_{1} \dot{e_{1}}+e_{2} \dot{e_{2}}+e_{3} \dot{e_{3}} \\
& =e_{1}\left(a x_{2}-a \beta_{1} y_{2}-a e_{1}-\beta_{1} u_{1}\right)+e_{2} x_{1} x_{3}-e_{2}\left(\beta_{2} y_{1} y_{3}\right. \\
& \left.+\beta_{2} u_{2}\right)+e_{3}\left(50\left(1-\beta_{3}\right)-b\left(x_{1}^{4}-\beta_{3} y_{1}^{4}\right)-c e_{3}-\beta_{3} u_{3}\right)
\end{aligned}
$$

Substitute equation (11) into equation (13)

$$
\begin{aligned}
& \dot{V}(e)=e_{1}\left(-e_{1}\right)+e_{2}\left(-e_{2}\right)+e_{3}\left(-e_{3}\right) \\
& \dot{V}(e)=-\left(e_{1}^{2}+e_{2}^{2}+e_{3}^{2}\right) \\
& \dot{V}(e)<0
\end{aligned}
$$

\subsection{Projective synchronization with the different scal- ing factors}

In this section, we present projective synchronization with differ-
Therefore, the error dynamical system (10) is asymptotically stable at the origin according to the Lyapunov stability theory. And the drive system (1) and the response system (2) can approach projective synchronization with different scaling factors asymptotically with the controllers (11). The proof is now complete. 


\subsubsection{Result discussion and output of results}

In order to verify the effectiveness of the proposed controllers in (11), numerical simulations are performed. Choose the scaling matrix $\beta=\operatorname{dia}[1,3,5]$ the 4 th order Runge-Kutta method is employed to integrate the differential equations. The initial conditions of the drive system and the response system are $(2.6,3.7,1.4)$ and $(1.4,5.9,2.1)$, respectively. Figure 4 shows that the variable errors do not synchronize with time in the absence of controllers but when the controllers are activated at $t \geq 5.5$ although the initial conditions are different. The state variables of the system (1) are in proportion to that of the system (2) with different scaling factors $1,3,5$, respectively. This is confirmed by the synchronization quality $e=\sqrt{\left(e_{1}^{2}+e_{2}^{2}+e_{3}^{2}\right)}$, given by as shown in Figure 5 .

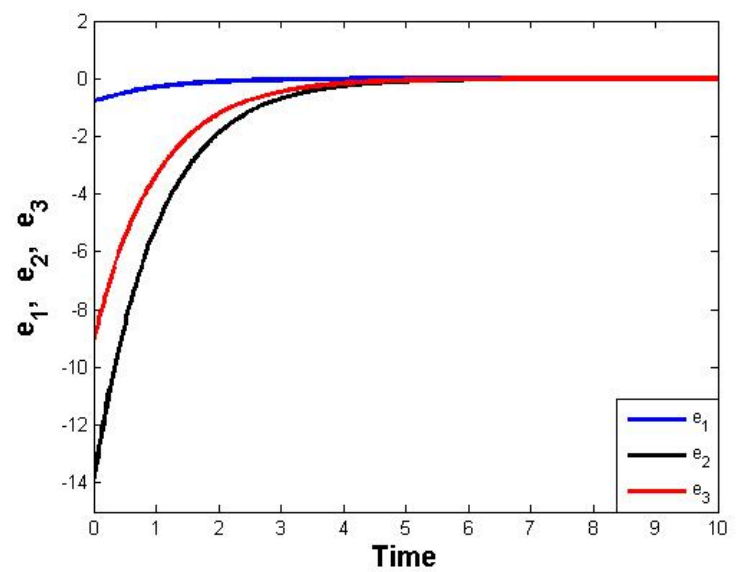

Figure 4: Error dynamics between systems (1) and (2) with the controllers deactivated for $0<t=5.5$ and activated for $t \geqslant 5.5$.

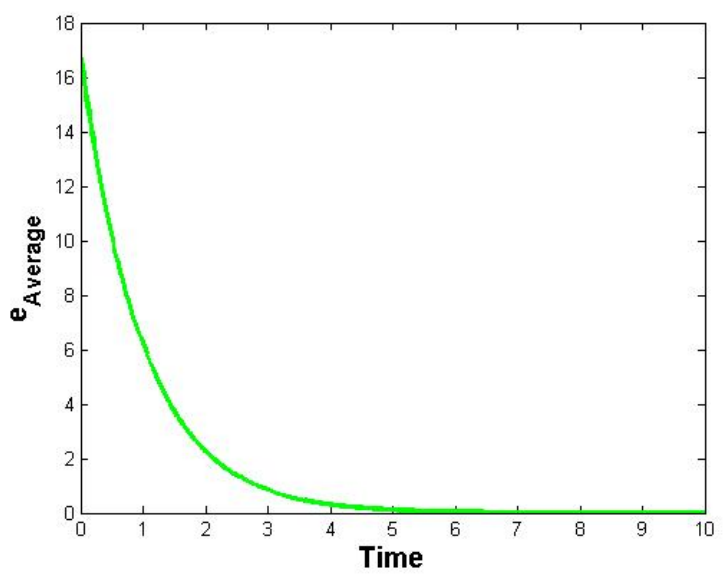

Figure 5: Synchronization quality between systems(1) and (2).

\section{CONCLUSION}

In this research work, we have observed projective synchronization behaviour of two identical new three-dimensional system with two nonlinearities equation (1). The conservative chaotic systems have the important property that they are volume conserving. Generaly, The Kaplan-Yorke dimension is calculated from $D_{K Y}=2+\frac{L_{1}+L_{2}}{\left|L_{3}\right|}$ for equation (1) and it is found to be 2.8554 The high value of the Kaplan-Yorke dimension indicates the complexity of the chaotic system. The Lyapunov exponents of the 3-D novel chaotic system have been obtained as $L_{1}=-1.0000, L_{2}=-10.8352$ and $L_{3}=$ 13.8356. Also, the maximal Lyapunov exponent of the 3-D novel conservative chaotic system is $L_{3}=13.8356$. The phase portraits, figure (1) of the novel chaotic system were simulated using MATLAB. Generalized projective synchronization is a general type of synchronization which generalizes common types of synchronization such as complete synchronization (CS), anti-synchronization (AS), hybrid synchronization (HS), projective synchronization (PS), etc. The PS synchronization result was established using Lyapunov stability theory. Finally, we have investigated projective synchronization behaviour of two identical new three-dimensional system with two nonlinearities with the same and different scaling factors. The results are validated by numerical simulations using MATLAB. It has more advantage over other synchronization to enhance security of communications function projective synchronization is more unpredictable and moreover it is performed for hyperchaotic system, which makes it more useful.

\section{AUTHORS' CONTRIBUTIONS}

Babatunde A. Idowu - developed the statement of the problem, the route to follow as well as necessary tools to utilize, collated all the different sections, their arrangements and also proof read with major emphasis on the introduction and necessary references. The expanded abstract and conclusion was developed with Cornelius O. Ogabi. Kehinde S. Oyeleke and Olasunkanmi I. Olusola - did the analytical calculations as well as the numerical simulations whilst Cornelius O. Ogabi wrote the introduction, re-confirm the analytical calculations and the numerical simulations and replotting where necessary.

\section{CONSENT (WHERE EVER APPLICABLE)}

Consent form has been approved by all authors. 


\section{REFERENCES}

[1] Lorenz, E. N, Deterministic non-periodic flow, Journal. Atmos. Sci. 1963; 20: 130-141.

[2] Lu ,J.H and Chen, R A new chaotic attractor coined., Int. J. Bifurc. Chaos. 2002; 3: 659-661 .

[3] Leonov G. A. ,. Kuznetsov N.V, On differences and similarities in the analysis of Lorenz, Chen, and Lu systems, Appl. Math. Comput.,2015; 256: 334-343.

[4] Zhang,F. C. Liao X. F. , Zhang G. Y. ,On the global boundedness of the Lü system, Appl. Math. Comput. 2016; 284: 332339.

[5] Chen G., Ueta T., Yet another chaotic attractor, Int. J. Bifurc. Chaos. 1999; 9: 1465-1466.

[6] Rossler O. E, An equation for hyperchaos, Phys. Lett. A 1979; 2(3): 155-157.

[7] Babatunde A. Idowu, Sundarapandian Vaidyanathan, Aceng Sambas, Olasunkanmi I. Olusola and O. S. Onma, A New Chaotic Finance System: Its Analysis, Control, Synchronization and Circuit Design, (2018);271-295 in (Nonlinear Dynamical Systems with Self-excited and Hidden Attractors, Studies in Systems, Decision and Control, edited by V. T. Pham et. al., Switzerland:Springer International Publishing)

[8] Hung M. L., J. J. Yan, T. L. Liao,Generalized projective synchronization of chaotic nonlinear gyros coupled with deadzone input, Chaos Soliton Fract. (2008); 35: 181-187.

[9] Luo T. Q., Z. Wang, Dynamics and SC-CNN circuit implementation of a periodically forced non-smooth mechanical system, Nonlinear Dyn. (2016); 85: 87-96.

[10] Xu Y. , H. Wang, Y. G. Li, et al.,Image encryption based on synchronization of fractional chaotic systems, Commun. Nonlin. Sci. Numer. Simul. (2014); 19: 3735-3744.

[11] Tong X. J., M. Zhang, Z. Wang, et al.,A joint color image encryption and compression scheme based on hyper-chaotic system, Nonlinear Dynamics.(2016) 84 2333-2356.

[12] Mata-Machuca J. L. , R. Martinez-Guerra, R. Aguilar-Lpezb, et al.A chaotic system in synchronization and secure communications, Commun. Nonlin. Sci. Numer. Simul. (2012); 17: 1706-1713.

[13] Zhang S, T. G. Gao, A coding and substitution frame based on hyper-chaotic systems for secure communication, Nonlinear Dynamics. 84 (2016) 833-849.

[14] Hassan M. F. , Synchronization of uncertain constrained hyperchaotic systems and chaos-based secure communications via a novel de-composed non-linear stochastic estimator, Nonlinear Dyn.(2016); 83: 2183-2211.

[15] L. M. Pecora and T. L. Carroll. Synchronization in chaotic systems. Phys-ical Review Letters, 1991; 64:821-824.
[16] Louis M. Pecora, Thomas L. Carroll, Gregg A. Johnson, Douglas J. Mar, and James F. Heagy. Fundamentals of synchronization in chaotic systems, concepts, and applications. Chaos, 1997; 7(4):520-543. Part of a special issue dedicated to synchronization of chaotic systems.

[17] Rybalova E. , N. Semenova,Transition from complete synchronization to spatio-temporal chaos in coupled chaotic systems with nonhyperbolic and hyperbolic attractors, Eur. Phys. J. Special Topics. (2017); 226: 1857-1866.

[18] Ma J. , F. Li, L. Huang, et al.,Complete synchronization, phase synchronization and parameters estimation in a realistic chaotic system, Commun. Nonlinear Sci. Numer. Simul. (2011); 16: 3770-3785.

[19] Yang J. Z. , G. Hu, Three types of generalized synchronization, Phys. Lett. A (2007); 361: 332-335.

[20] Vijay K, Yadav1, S. K. Agrawal2, et al.,Phase and antiphase synchronizations of fractional order hyperchaotic systems with uncertainties and external sturbances using nonlinear active control method, Int. J. Dynam.(2017); 5: 259-268.

[21] Liu H. M. , W. G. Sun,Ghada Al-mahbashi, Parameter identification based on lag synchronization via hybrid feedback control in uncertain drive-response dynamical networks, Adv. Differ. Equ-ny. (2017); 2017: 122.

[22] Li C. G., X. F. Liao, J. B. Yu,Synchronization of fractional order chaotic systems, Phys. Rev. E. (2003); 68: 067203.

[23] Lu J. G. ,Chaotic dynamics and synchronization of fractionalorder Arneodo's systems, Chaos Soliton Fract. (2005); 26: 1125-1133.

[24] Wang Q. Y. , Q. S. Lu, Z. S. Duan, Adaptive lag synchronization in coupled chaotic systems with unidirectional delay feedback, Int. J. Non-Linear Mech.(2010); 45: 640-646.

[25] Olusola, O. I., Idowu B. A., Vincent U. E and Odun$\tan$ A. E., Control, Projective Synchronization and AntiSynchronization of Hyperchaotic Lorenz Stenflo Systems, J. of Nigeria Association of Mathematical Physics,(2017); vol. 40 pp. $49-56$.

[26] Voss H. U. , Anticipating chaotic synchronization, Physical Review E. (2000); 61: 5115-5119.

[27] Park J. H.,Exponential synchronization of the Genesio-Tesi chaotic system via a novel feedback control, Phys. Scripta. (2007); 76: 617-622.

[28] Zhang Q. J. , J. A. Lu, Z. Jia,Global exponential projective synchronization and lag synchronization of hyper-chaotic Lü system, Commun. Theor. Phys. (2009); 51: 679-683.

[29] Tavazoei M. S., M. Haeri,Synchronization of chaotic fractional-order systems via active sliding mode controller, Physica A. (2008); 387: 57-70. 
[30] Li C. P., J. P.Yan, The synchronization of three fractional differential systems, Chaos Soliton Fract. (2007); 32: 751-757.

[31] Wang Y. ,Dynamic analysis and synchronization of conformable fractional-order chaotic systems, Eur. Phys. J. Plus (2018); 133: 481.

[32] WANG L. M.,Model-free adaptive sliding mode controller design for generalized projective synchronization of the fractional-order chaotic system via radial basis function neural networks, Pramana - J. Phys. (2017); 89: 38.

[33] Li, Guo-Hui. Modified projective synchronization of chaotic system. Chaos, Solitons and Fractals 32.5 (2007): 1786-1790.

[34] Li, Guo-Hui. Modified projective synchronization of chaotic system. Chaos, Solitons and Fractals 32.5 (2007): 1786-1790.

[35] Wang, Xingyuan, and Yijie He. Projective synchronization of fractional order chaotic system based on linear separation. Physics Letters A 372.4 (2008): 435-441.

[36] Vaidyanathan, S., and S. Pakiriswamy. A 3-D Novel Conservative Chaotic System and its Generalized Projective Synchronization via Adaptive Control. Journal of Engineering Science and Technology Review (2015); 8.2

[37] Shi-Quan, Shao, Gao Xin, and Liu Xing-Wen. Projective synchronization in coupled fractional order chaotic Rossler system and its control. Chinese Physics 16.9 (2007): 2612.

[38] Li, Chunguang. Projective synchronization in fractional order chaotic systems and its control. Progress of theoretical physics 115.3 (2006): 661-666.

[39] Chun-Lai, Li, et al. Projective synchronization for a fractional-order chaotic system via single sinusoidal coupling. Optik 127.5 (2016): 2830-2836.

[40] Chen, Diyi, et al. Synchronization between integer-order chaotic systems and a class of fractional-order chaotic systems via sliding mode control. Chaos: An Interdisciplinary Journal of Nonlinear Science 22.2 (2012): 023130.

[41] Li, G., Zhous S P., Yang K. Generalized Synchronization between two different chaotic systems using active backstepping control. Phys. Lett. A.(2006); 355: 326 - 330.

[42] Jia Qiang. Projective Synchronization of a new hyperchaotic Lorenz System. Phys. Lett. A. (2007); 370: 40-45.

[43] Boulkroune, Abdesselem, Amel Bouzeriba, and Toufik Bouden. Fuzzy generalized projective synchronization of incommensurate fractional-order chaotic systems. Neurocomputing 173 (2016): 606-614.

[44] Idowu, B. A., Vincent, U. E. Synchronization and Stabilization of Chaotic Dynamics in a Quasi-1D Bose-Einstein Condensate, Journal of Chaos, (2013); Vol. 2013,Issue 723581,Pp. 1-8
[45] Idowu, B. A., Rongwei Guo, Vincent, U. E. Adaptive Control for the Stabilization and Synchronization of Nonlinear Gyroscopes, Int. J of Chaos, Control, Modelling and Simulation,(2013); Vol. 2,Issue 2,Pp. 27-44.

[46] Idowu, B. A., Vincent, U. E, Njah, A. N. Synchronization of Chaos in Non-identical Parametrically Excited Systems, Chaos Solitons and Fractals,(2009); Vol. 39,Issue ,Pp. 23222331 .

[47] Guo, R., Vincent, U. E., Idowu, B. A. Synchronization of chaos in RCL-shunted Josephson junction using a simple adaptive controller, Physica Scripta,(2009); Vol. 79,Issue 035801,Pp. 1-6 .

[48] C. O. Ogabi, B. A. Idowu, A. S. Ogungbe, E. O. Somoye, E. O. Onori, O. O. Ometan, Rasaq Adeniji-Adele, A. Ogwala, Euler's Equations of Rigid Body: Its Chaos Control, Tracking and Synchronization, Journal of Resesrch and Review in Science,(2018); Vol. 5 pp $146-152$

[49] Vaidyanathan, S., K. Madhavan and B. A. Idowu, Backstepping Control Design for the adaptive stabilization and synchronization of the Pandey Jerk Chaotic System with Unknown Parameters, Int. Journal of Control Theory and Applications,(2016); Vol. 9,Issue 1,Pp. 299-319

[50] S. Vaidyanathan, B. A. Idowu and A. T. Azar. Backstepping Controller for the Global Chaos Synchronization of Sprott's Jerk Systems, (2015); 39-58 in Chaos Modeling and Control Systems Design, Studies in Computational Intelligence 581, edited by Azar, Ahmad Taher, Vaidyanathan, Sundarapandian, Switzerland:Springer International Publishing

[51] Vaidyanathan S., Volos Ch., Pham V-T., Madhavan K., Idowu, B. A., (2014), Adaptive Backstepping Control, Synchronization and Circuit Simulation of a 3-D novel Jerk Chaotic System with Two Hyperbolic Sinusoidal Nonlinearities, Archives of Control Sciences, Vol. 24,Issue 3,Pp. 257-285

[52] Idowu, B. A., Vincent, U. E, Njah, A. N., (2009), Generalized Adaptive Backstepping Synchronization for Nonidentical Parametrically Excited Systems, Nonlinear Analysis: Modelling and Control, Vol. 14,Issue 2,Pp. 165-176

[53] Idowu, B. A., Vincent, U. E, Njah, A. N., (2008), Control and Synchronization of Chaos in Nonlinear Gyros via Backstepping Design, Int. Journal of Nonlinear Science, Vol. 5,Issue 1,Pp. 11-19

[54] Vaidyanathan S., and B. A. Idowu, (2016), Adaptive Control and Synchronization of Chlouverakis-Spott Hyperjerk System via Backstepping Control, 117-142 in Advances and Applications in Nonlinear Control Systems, Studies in Computation Intelligence 635 , edited by S. Vaidyanathan and C. Volos,Switzerland:Springer International Publishing.

[55] Vaidyanatan S., Abba O. A., Betchewe G., and Alidou M. A new three-dimensional chaotic system: its adaptive control and circuit design. Int. J. Automation and Control,; (2019); 13(1): $100-121$. 
[56] Xu, Daolin, and Zhigang Li. Controlled projective synchronization in nonpartially-linear chaotic systems. International Journal of Bifurcation and Chaos 12.06 (2002): 1395-1402.

[57] Strogatz, S.H.. Nonlinear Dynamics and Chaos: with applications to Physics, Biology, Chemistry and Engineering. Perseus Books, U.S.A.(1994)

[58] Park, Ju H. ,Adaptive modified projective synchronization of a unified chaotic system with an uncertain parameter Chaos Solitons Fractals (2007); 34(5) : 1552-1559.

[59] Cai, Na, Yuanwei Jing, and Siying Zhang. Modified projective synchronization of chaotic systems with disturbances via active sliding mode control. Communications in Nonlinear Science and Numerical Simulation 15.6 (2010): 1613-1620.

[60] Li, Guo-Hui. Projective Synchronization of Chaotic System using backstepping control. Chaos Soliton and Fractals (2006); 29: 490-494. 